\title{
THE MANAGEMENT OF THE BLADDER IN SPINAL PARAPLEGIA
}

\author{
T. COETZEE, M.B., Ch.B., Ch.M., F.R.C.S.(Eng.), F.C.S. (S.A.), F.A.C.S.
}

\author{
Lecture delivered to the S.A.S.P. Post Registration Course \\ at the \\ Ernest Oppenheimer Hospital, Welkom, May, 1965.
}

The paraplegic and the care of the paraplegic bladder is a world-wide problem. In some cases the condition is the result of disease; mostly it is man-made. With the advance of industrialization and the increase in road accidents it can be safely assumed, unfortunately, that the number of these patients will increase in the future. In this hospital our patients with paraplegia are mostly the victims of industrial accidents: a fracture of the spine with injury of the spinal cord and/or cauda equina. In a minority of cases paraplegia is the result of spinal tuberculosis or tumour. However, whether the paraplegia has resulted from trauma or disease of the spine or spinal cord, the problems presented by the bladders are similar.

Part of what I shall be saying today is possibly not the direct concern of the physiotherapist. However, it is my experience that sharply divided responsibilities in the care of the paraplegic reflects usually to the detriment of the patient. The surgeon, the nurse, the physiotherapist, the appliance expert, the hospital almoner and social workerall these should work as a team if the most good is to be done for the patient. If this is so, then it also follows that the knowledge and experience of these various disciplines should be pooled and each should have a working acquaintance with the methods of the others. I am therefore making no excuses for mentioning certain facts which may not be directly or primarily the province of the physiotherapist.

If you will bear with me for a few minutes, I shall briefly present the anatomy and physiology necessary for the understanding of the cord bladder.

In the adult the spinal cord occupies the vertebral canal as far down as the lower border of the first lumbar vertebra. In the conus medullaris - the tapering caudal extremity of the spinal cord, all the sacral segments of the cord are crowded into the part of the cord lying opposite the first lumbar vertebra. Below this level, injury will result in pure cauda equina, i.e. nerve root damage, above this level injury may be isolated cord damage or cord plus root damage. The differentiation is most important: injury to the spinal cord is irrecoverable, while regeneration is possible with root damage.

Above the conus level (for practical purposes down to $D$ 11) the sacral segments of the cord are undamaged, spinal reflex paths remain intact; but cortical control is interrupted.

The motor nerves of the bladder are the parasympathetic nerves contained in the $2 \mathrm{nd}, 3 \mathrm{rd}$ and the 4 th sacral nerves. These, as parasympathetic nerves do, end in a peripheral plexus or actually in the bladder wall from where postganglionic fibres are given to the bladder muscle. The external urethral sphincter is also supplied from the same spinal segments via the pudenal nerve.

Urinary continence is usually acquired by the child before the age of two years: this implies the awareness of the sensation of fullness of the bladder, and the ability to postpone the act of urination until the time and place is opportune. The bladder muscle-the detrusor-possesses, to a remarkable degree, the quality of accommodation and the sensation of fullness will pass away temporarily if urination is not possible. It will, however, return, more urgently as filling proceeds, until the sensation becomes acutely painful.

Urination consists in the voluntary removal of inhibition

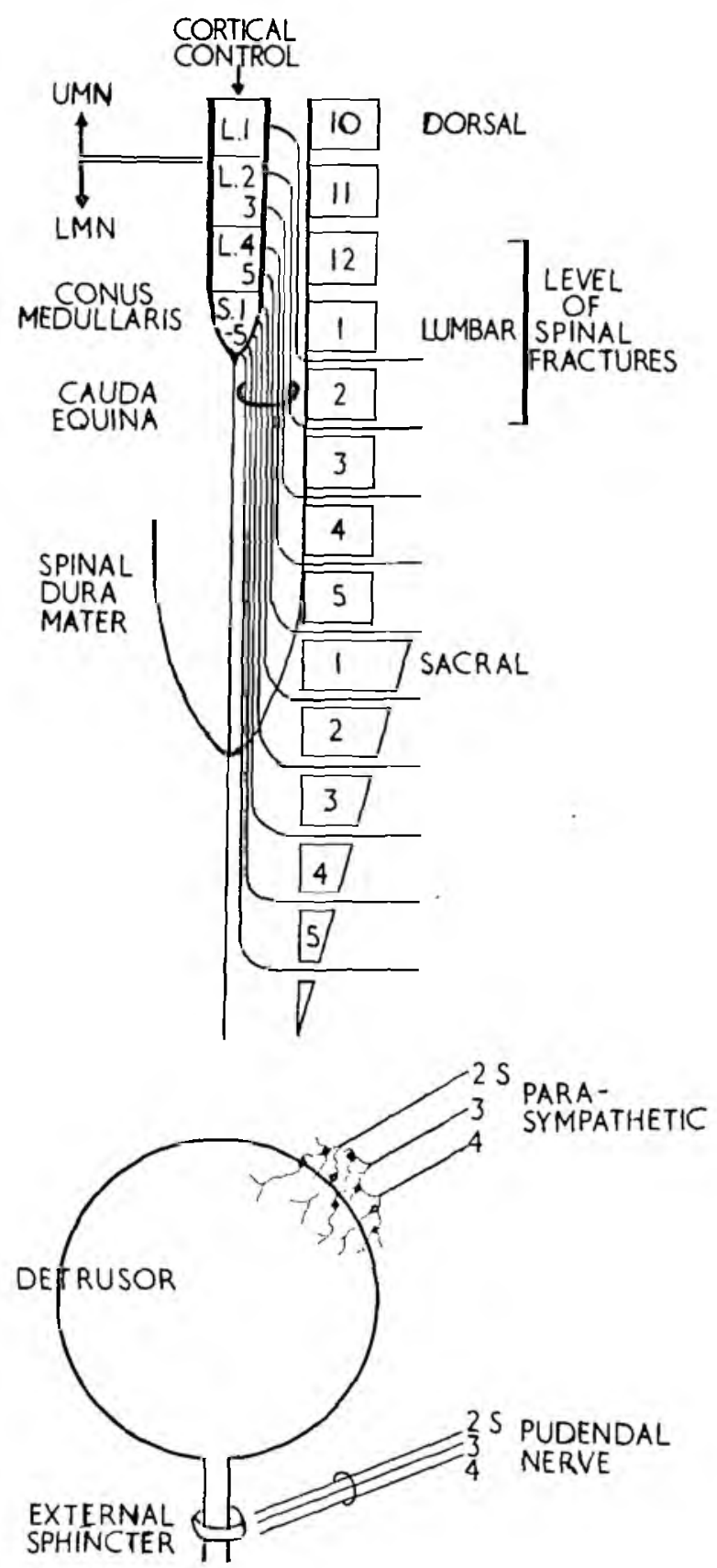


of the sacral spinal segments, leading to parasympathetic discharge, contraction of the detrusor with opening of the bladder neck and outflow of urine. The external sphincter is merely a cut-off muscle on guard during stress or for voluntary interruption of the urinary stream. Normally continence is maintained by urethral resistance, the result of the tone of its muscular walls.

When spinal cord injury involves the sacral segments of the cord, reflex urination is interfered with or abolished. The bladder has to be emptied by abdominal straining or manual compression. The patient often has a vague sensation of bladder fullness. Para-sympathetic discharge will occur, increasingly with time, from plexuses close to or in the bladder wall, to the extent that hypertrophy of the bladder muscle and bladder neck may occur. This has important consequences: the bladder wall thickens to the extent that its capacity is seriously reduced and obstruction develops at the bladder neck. The patient usually dribbles or passes small amounts of urine at frequent intervals and the residual urine is considerable. The best that can usually be achieved is total leak with a small residual, controlled with an external appliance. This type of cord bladder is common as vertebral level T 12-L 2 is the most frequent site of spinal injury.

After injury at higher levels reflex bladder emptying will be retained. The bladder empties, involuntarily, when a certain degree of filling has occurred. Emptying can sometimes be triggered by pressing over the hypogastrium, by scratching the inside of the thigh or squeezing the glans penis. This type of cord bladder has often been regarded, in the past, as a happy state of affairs. However, bladder emptying is often incomplete, intervals of dryness often short and unpredictable and there is often deterioration of bladder function with time due to increased spasticity of the urethral and pelvic floor musculature.

In some of these cases successful bladder emptying with retention of reflex urination can be achieved by relieving bladder neck and urethral resistance, either by bladder neck resection or pudendal neurectomy.

In all cases of spinal cord injury there will be, during an initial phase of varying duration, complete flaccidity of the bladder with retention of urine.

In all cases the aims of treatment are:

A. To avoid overdistension of the bladder during the initial phase of complete retention: It should be remembered that secretion of urine is usually diminished in the immediate post-traumatic phase. Relief of urinary retention is therefore never dreadfully urgent. For instance, there is no need for a catheter to be passed at the scene of an accident or at the dressing station or hospital outpatient department. This is sometimes done and infection is often introduced when proper aseptic technique is not possible or observed. Catheterization should be postponed until it can be performed, under ideal conditions, in the ward or theatre.

Many different techniques for managing the bladder during this phase of retention have been recommended. This varies from overflow plus expression, to suprapubic cystostomy, intermittent catheterization, perineal urethrostomy and an indwelling urethral catheter-with simple drainage, regular bladder irrigation or tidal drainage.

Our personal preference is very definitely for indwelling catheter drainage, using for this purpose a $16 \mathrm{~F}$ latex rubber catheter with a retaining bulb draining into a container with a water seal. Our experience with Gibbon and other plastic catheters has not been satisfactory. Routine bladder irrigations are not used but may sometimes be required, temporarily, in the presence of urinary infection. To avoid kinking and pressure at the peno-scrotal area, the catheter and penis is draped upwards and outwards over the lower abdomen. The catheter-penis junction is covered with gauze moistened in antiseptic. Drainage tubing and receptacles are sterilized daily, observing aseptic precautions during removal and re-connection. The catheter is changed when necessary, using a new catheter every time. We like to have the pubic hair shaved-local cleanliness is made easier.
B. To restore bladder emptying or improve its efficiency: Resumption of detrusor activity can generally first be observed during cystometography. More usually, the urethral catheter is simply removed and bladder function assessed by noting the result of voiding efforts, the occurrence of spontaneous leakage, the result of expression and measurement of the residual urine.

In our own work, a residual urine of more than $100 \mathrm{ml}$. is regarded as too risky to accept. In such cases drainage will be reinstituted. It is most important not to permit overdistension of the bladder during such a check-up. Ascending urinary infection has often started at this stage.

If sufficient bladder emptying has not been restored at three months following trauma, further investigations are instituted. The most instructive investigation is the voiding cystogram-this will usually show the presence of abnormal resistance and its site and will indicate the nature of any operative procedures that may be necessary.

C. To control incontinence: In the male the most convenient method is by using an external appliance. These vary in design but the object is to collect the urine from the penis by means of a cuff with a water-tight fit and drain it into a collecting bag. In this way the patient may be kept dry and could attend to his daily task. The main difficulty is to avoid maceration and ulceration of the penile skin, the glans penis and the external urethral meatus. Some male patients prefer an indwelling catheter and may tolerate it well for many years.

It is amazing what insults the urinary tract will sometimes tolerate. Some years ago I had the opportinity of interviewing an old gentleman who attended in the outpatient department of the hospital where I was then working. He had been catheterizing himself ever since an attack of acute urinary retention and his only reason for attending was to obtain a new catheter. I took him to an examination room and asked him to demonstrate his technique to me. He took, from his wallet in an inside pocket, an old battered and scratched coudé gum-elastic catheter, wiped it with his handkerchief, applied a liberal dash of saliva, directly, as a lubricant, and passed the instrument into the bladder. He had been doing this, using exactly the same technique, roughly four times a day, for 25 years. There was no evidence of urinary infection. When I warned him of the dangers of infection he explained that he chewed tobacco and did not think that any organism could exist in that environment. . .

On other occasions a single, scrupulously aseptic, urethral catheterization will spark off a dangerous ascending urinary infection.

No suitable external appliance for the incontinent female is available as yet and a permanent indwelling catheter is usually the besi solution.

In some neglected cases with severe bladder contractions, upper tract dilatation and deteriorating renal function it may be necessary to construct an ileal conduit. With an efficient ileostomy appliance this can be made to work very well.

Several other aspects of the care of the paraplegic patient has a close bearing on the management of his bladder:

1. The prevention of pressure sores: In this hospital we are fortunate in almost never seeing a pressure sore-so much so that we almost welcome the admission of a patient with a pressure sore once in a while to be able to show our nursing staff why we insist on two-hourly turning of the patient. The prevention of pressure sores is, in the acute phase, the responsibility of the nursing staff. At a later stage, with the patient in his wheelchair, the supervision by the physiotherapist and the co-operation of the patient are the most important safeguards. Vigilance, in this respect, must continue for life.

2. The prevention of urinary sepsis: This is without doubt the most important aspect of the case of the paraplegic as far as life expectancy is concerned. But apart from this it has immediate consequences of great importance. Any sepsis introduced will alter the whole sequence of the evolution of the cord bladder and will most often result in a contracted 
bladder with incontinence and upper tract dilatation, infection and formation of calculi in the kidneys or bladder. But, in addition, the infection (with that present as a result of pressure sores) will alter the whole behaviour of the nervous system resulting for example in more severe flexor spasms.

The problem of urinary sepsis is a permanent one. The best method of prevention is to secure good emptying of the bladder as early as possible and to check up that this is maintained.

3. The occurrence of flexor spasm: This complicates bladder care. Hip flexion will make local manipulations difficult and flexor spasms will often trigger bladder emptying. It is therefore most important to maintain a full range of passive movement in the patient's lower extremities.

4. Priapism: This is not often severe. It is usually more prominent in the higher lesions. It is sometimes awkward when it occurs during cystoscopy, and urethritis is more frequent as elongation of the organ will cause the unsterile part of an indwelling catheter to slide into the urethra.

5. The care of the bowels: This is a most important aspect of the management of the paraplegic bladder. It has been repeatedly observed that a loaded rectum impairs the patient's ability to empty his bladder. The care of the bowel differs considerably in various clinics: in this hospital it was customary to administer a soap enema every second or third day. This was followed by a rectal examination to check on complete clearance and digital evacuation if necessary. A regular evening aperient was given-the most popular being milk of magnesia with paraffin or senokot. A high-bulk breakfast food was tried without any noticeable improvement.

Frequent digital evacuation may cause excoriation of the anal canal and perianal abscesses may then form-these are very slow to resolve.

At present we use a contact-action suppository every second day and this appears to work well. It is a procedure which can easily be performed by the patient after leaving hospital.

6. Lastly-I wish to refer only briefly to a most important aspect of the care of the paraplegic-I am referring to his emotional rehabilitation.

What it must mean for a young healthy adult to discover, after an accident, that the lower half of his body is dead and to be told, as he must be told, that the condition will be permanent, is known only to those who have actually had the experience. It is the responsibility of all those who have to attend to such a patient, to assist in persuading him to accept the condition, but, at the same time, to reassure him that all is not lost and that, at least, an interesting and useful life is still possible. It is only when this healthy emotional state has been achieved that physical rehabilitation will make progress. A patient who is not interested in even continuing to live, will certainly not show any interest in attending to the emptying of his bladder. I mention this particular aspect because it ties up with our main theme today.

I hope that these and other points will be raised during the discussion and it will be very interesting to hear what is being done in other clinics.

In conclusion I wish to mention a few lines of research which are now being actively pursued:

Evacuation of the paraplegic bladder by electrical stimulation has been attempted and the techniques have been used in the human patient. There are two methods: In the first method electrodes are placed on the bladder wall with the connecting wires coming through the skin and these are connected to a source of electrical stimuli.

The second method uses similar electrodes and these are connected to a small receiving apparatus which is implanted just deep to the skin of the hypogastrium. A transmitter is employed and this is held close to abdomen skin or its aerial is placed near the body. The receiver picks up the transmitted waves and these are conducted to the stimulating electrodes on the bladder wall. The type of wave form its frequency and duration of application, the voltage, the placement of the electrodes and their composition-all these have been worked out in great detail.

The main trouble with these methods have been infection, intolerable pain or stimulation, and spread of the stimulus especially to the muscles of the pelvic floor and perineum.

Successful results have been obtained and further experimental work is proceeding.

A completely different approach is to excise the whole paraplegic bladder, leaving only the bladder neck and to replace it with a substitute fashioned from an isolated loop of ileum. The ileum retains its parasympathetic nerve supply (i.e. the vagus nerve) intact. Emptying of this new pouch, on stimulation of the vagus, has been observed.

A method which has been used to overcome incontinence will finally be mentioned. It also uses a transmitter and an implanted receiver as previously described but the electrodes are placed on each side of the external urethral sphincter. As long as the transmission lasts the sphincter will remain contracted. By manipulation of a switch evacuation of the bladder can be started.

It seems appropriate, in our machine age, that a patient who has wetted his bed, could possibly explain it by saying: "Oh, my battery failed". I can imagine similar machine-age aids being used for other physiological deficiencies with even more disturbing results following "battery" failure.

\section{WORLD CONFEDERATION FOR PHYSICAL THERAPY : \\ FIFTH INTERNATIONAL CONGRESS \\ Melbourne, 15th to 26th May, 1967 \\ "MODERN APPROACH TO ANCIENT ILLS" \\ Preliminary Programme}

Monday, 15th May

Morning:

Registration.

Opening of Congress.

Opening of Trade Exhibition.

Opening Lecture.

\section{Afternoon:}

"Spina Bifida," including lectures by a neurosurgeon, an orthopaedic surgeon, and a physiotherapist, followed by a demonstration.

Visits to Spina Bifida Clinic, Royal Children's Hospital, and Yooralla Hospital School.

\section{Morning:}

Tuesday, 16th May

"Rehabilitation of Head Injuries," including lectures on a specific brain condition by a surgeon, general considerations by a physiotherapist, and a "Symposium on the Rehabilitation of the Patient with Head Injuries" by a team of lecturers including surgeon, physiotherapist, speech therapist, psychiatrist, and occupational therapist, followed by discussion groups and demonstration.

Visits to Austin Hospital, St. Vincent's Hospital, and Hampton and Coonac Rehabilitation Centres.

\section{Afternoon:}

"Sports Medicine," with a lecture on physiology of exercise by a physiologist, and a Symposium by a sports medicine specialist, an athlete doctor, a coach, and a physiotherapist, with a demonstration on "Strapping" by a physiotherapist, followed by discussion groups.

Special Interest Group. 